\title{
The detection of heavy metals in the circumstellar envelopes of post-AGB stars
}

\author{
V.G. Klochkova ${ }^{1}$ \\ ${ }^{1}$ Special Astrophysical Observatory, Nizhnij Arkhyz, 369167 Russia \\ (August 15, 2021)
}

\begin{abstract}
A new type of peculiarity - a splitting or asymmetry of strong absorption lines, is found in the optical spectra of selected post-AGB stars with C-rich circumstellar envelopes. The effect is maximal in Ba II lines whose profile is split into two-three components. The particular components of the split absorption lines are shown to be formed in a structured circumstellar envelope, suggesting an efficient dredge-up of the heavy metals produced during the preceding evolution of this star into the envelope. We suspect that the splitting (or asymmetry) of the profiles of strongest absorptions with low excitation potential of the low level can be associated with the kinematic and chemical properties of the circumstellar environment and with type of its morphology.
\end{abstract}

\section{INTRODUCTION}

Now generally accepted that about half of abundances of chemical elements heavier than iron are produced by slow neutron captures (s-process) in the deep layers of asymptotic giant branch (AGB) stars. Mixing brings freshly synthesized heavy elements to the stellar surface (the third dredge-up process) and then stellar wind carries them away in the stellar enviroments. This is a simplified description of the processes, the sequence of which enriches both circumstellar and interstellar medium with heavy metals. For more detail see, e.g., [1, 2] and references there. AGB stars are therefore the principal suppliers of heavy metals and important suppliers of carbon and nitrogen to the interstellar medium, thereby participating in the chemical evolution of galaxies. In this article, we shall focus on the first observational evidences on the presence of circumstellar spectral features of heavy metals in the optical spectra of nearest descendants of AGB-stars-protoplanetary nebulae (PPNe).

PPNe are objects at the post-asymptotic branch (post-AGB) stage of evolution. These descendants of AGB stars are low-mass cores with typical masses of $0.6 \mathrm{M}_{\odot}$ surrounded by an extended and often 
structured gaseous-dusty envelope, which formed as a result of substantial mass loss by the star during the preceding evolutionary stages. Central stars are usually surrounded by envelopes in the form of extended haloes, arcs, lobes, and tori. Some stars exhibit various combinations of the above features as well as bipolar and quadrupolar nebulae with dust bars. Examples of the latter two types include the Egg nebula = RAFGL 2688 and IRAS 19475+3119 nebulae, for which high-resolution images were taken by the Hubble Space Telescope [3]. All PPNe and about $80 \%$ of planetary nebulae are asymmetric [4]. Much remains to be understood about condensation of dust particles and formation of the dust fraction in AGB star envelopes (see [5] and references therein).

A circumstellar gas and dust envelope shows up in the form of peculiarities in the IR, radio, and optical spectra of post-AGB supergiants. The optical spectra of PPNe differ from those of classical massive supergiants by the presence of molecular bands superimposed onto the spectrum of an F-G supergiant and by the anomalous behavior of the profiles of selected spectral features. These may include complex emission and absorption profiles of HI, Na I, and He I lines, and metal emission features (see for detail [8] and references therein). Furthermore, all these peculiarities are variable. In whole we see that the known types of spectral features in the optical spectra of post-AGB stars are: 1) low- or moderate-intensity symmetric metal absorptions without apparent distortions; 2) complex profiles of neutral hydrogen lines, which vary with time and include absorption and emission components; 3) absorption or emission bands of mostly carbon containing molecules; 4) envelope components of the $\mathrm{NaI}$ and K I resonance lines, and also 5) narrow permitted or forbidden metal emission lines that form in envelopes. The presence of type 2-5 features is the key difference of the spectra of PPNe from those of massive supergiants.

Here we analyze the manifestations of circumstellar envelopes in the optical spectra of PPNe focusing on the homogeneous subsample of stars whose atmospheres, according to previous studies, underwent evolutionary variations of the chemical composition. Section 2 briefly describes the employed observational data and lists the studied stars and their basic parameters. In Section 3 we analyze the available data on the peculiarities of the profiles of metal lines, found in high-resolution spectra, as well as data on the presence of molecular bands and outflow velocities for objects with different envelope structures. In Sections 4 and 5 we discuss the obtained results and summarize the main conclusions.

\section{OBSERVATIONAL DATA}

Over the past two decades more than 40 post-AGB candidates - supergiants with IR excesses and several related luminous stars with unclear evolutionary status have been spectroscopically monitored with the 
6-m telescope of the Special Astrophysical Observatory. As a result, a collection of high-quality spectra has been acquired with the primary purpose of searching for anomalies of stellar chemical composition due to the nucleosynthesis of chemical elements in the interiors of low- and intermediate-mass stars and the subsequent dredge-up of the synthesis products to the surface layers of stellar atmospheres. These observational data are also used to search for peculiarities in the PPNe spectra, to analyze the velocity fields in the atmospheres and envelopes of these stars with mass loss, and to search for the likely long-term spectral and radial velocity pattern variations.

Here we use the data acquired in the Nasmyth focus with the NES [6] echelle spectrograph. The NES spectrograph, equipped with a $2048 \times 2048$ CCD and an image slicer, produces a spectroscopic resolution of $\mathrm{R} \approx 60000$. Since 2011 the NES spectrograph has been equipped with a $2048 \times 4096 \mathrm{CCD}$ which made it possible to significantly extend the wavelength coverage. The spectra of the faintest program objects (the optical component of the IR sources IRAS04296+3429 and $20000+3239$ ) were acquired with the PFES echelle spectrograph mounted in the primary focus of the 6-m telescope [7]. This spectrograph, equipped with a $1 \mathrm{k} \times 1 \mathrm{k} C \mathrm{CD}$, produces a spectroscopic resolution of $\mathrm{R} \approx 15000$. We described the details of spectrophotometric and position measurements of the spectra in our earlier papers, the corresponding references can be found in the original papers listed in the paper by [8].

\section{MAIN PECULIARITIES IN THE OPTICAL SPECTRA OF POST-AGB STARS}

Our comprehensive study of the program stars allowed us to determine (or refine) their evolutionary status. One of the results of our analysis is that the studied sample of luminous stars with IR excesses is not homogeneous [17]. In this paper we consider the peculiarities of the optical spectra of post-AGB stars paying special attention to the subsample of objects listed in Table 1. It contains IRAS objects with central stars whose atmospheres are overabundant in carbon and heavy metals. Their circumstellar envelopes have a complex morphology and are usually rich in carbon, as evidenced by the presence of $\mathrm{C}_{2}$, $\mathrm{C}_{3}$, CN, CO, etc. molecular bands in their IR, radio, and optical spectra. Presence and type of $\mathrm{C}_{2}$ bands for stars in Table 1 are published by [18-21]; [22, 24, 26-29].

Furthermore, the objects from Table 1 are among those few PPNe whose IR-spectra exhibit the so far unidentified emission band at $21 \mu \mathrm{m}$ [30, 31]. Despite an extensive search for appropriate chemical agents, so far no conclusive identification has been proposed for this rarely observed feature. However, its very presence in the spectra of PPNe with carbon enriched envelopes suggests that this emission may be due to the presence of a complex carbon-containing molecule in the envelope (see [31, 32] for details and 
Table 1. Basic data for $\mathrm{C}$-rich circumstellar envelopes of post-AGB stars. Details concerning the $\mathrm{C}_{2}$ bands in the third column see in the text. The last column gives the expansion velocity of the envelope as determined from the position of

Ba II circumstellar components.

\begin{tabular}{|c|c|c|c|c|c|}
\hline \multirow[t]{2}{*}{ Object } & \multirow{2}{*}{$\begin{array}{l}\text { Morphology } \\
\text { of the } \\
\text { envelope }^{a}\end{array}$} & \multirow{2}{*}{$\begin{array}{l}\text { Type of } \\
\text { the } \mathrm{C}_{2} \\
\text { bands }\end{array}$} & \multicolumn{3}{|c|}{$V_{\text {exp }}, \mathrm{km} \mathrm{s}^{-1}$} \\
\hline & & & $\mathrm{CO}$ & $\mathrm{C}_{2}$ & BaII \\
\hline $04296+3429$ & $\begin{array}{l}\text { bipolar }+ \\
\text { halo + bar }\end{array}$ & $\begin{array}{l}\text { abs } \\
\text { emis }\end{array}$ & $10.8^{b}$ & $\begin{array}{l}7.7^{g} \\
12^{h}\end{array}$ & \\
\hline $07134+1005$ & $\begin{array}{l}\text { elongated } \\
\text { halo }\end{array}$ & abs & $10.2^{b}$ & $\begin{array}{l}8.3^{g} \\
11^{i}\end{array}$ & \\
\hline $08005-2356$ & bipolar & $\begin{array}{l}\text { uncertain } \\
\text { abs }\end{array}$ & $100:^{c}$ & $\begin{array}{l}43.7^{g} \\
42 j\end{array}$ & \\
\hline $19500-1709$ & bipolar & no & $\begin{array}{l}17.2,29.5^{b} \\
10,30-40^{d}\end{array}$ & & $20 \& 30^{k}$ \\
\hline $20000+3239$ & $\begin{array}{l}\text { elongated } \\
\text { halo }\end{array}$ & 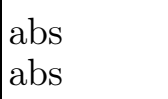 & $12.0^{e}$ & $\begin{array}{l}12.8^{g} \\
11.1^{l}\end{array}$ & \\
\hline RAFGL 2688 & $\begin{array}{l}\text { multipolar }+ \\
\text { halo }+ \text { arcs }\end{array}$ & $\begin{array}{l}\text { abs } \\
\text { emis }\end{array}$ & $17.9,19.7^{f}$ & $\begin{array}{l}17.3^{g} \\
60^{m}\end{array}$ & \\
\hline $22223+4327$ & $\begin{array}{l}\text { halo }+ \\
\text { small lobes }\end{array}$ & $\begin{array}{l}\text { abs. } \\
\text { emis }\end{array}$ & $14-15^{f}$ & $\begin{array}{l}15.0^{g} \\
15.2^{n}\end{array}$ & \\
\hline $22272+5435$ & $\begin{array}{l}\text { elongated }+ \\
\text { halo }+ \text { arcs }\end{array}$ & $\begin{array}{l}\mathrm{abs} \\
\mathrm{abs}\end{array}$ & $9.1-9.2^{b}$ & $\begin{array}{l}9.1^{g} \\
10.8^{o}\end{array}$ & $10^{\circ}$ \\
\hline $23304+6147$ & $\begin{array}{l}\text { quadrupole }+ \\
\text { halo }+ \text { arcs }\end{array}$ & $\begin{array}{l}\text { abs } \\
\text { emis }\end{array}$ & $9.2-10.3^{b}$ & $\begin{array}{l}13.9^{g} \\
15.5^{p}\end{array}$ & $15.1^{p}$ \\
\hline
\end{tabular}

Notes: a - morphology type of envelopes is taken from papers by [3, 4, 9, 10];

$b-[11], c-[12], d-[14], e-[15], f-[16], g-[18]$,

$h-[22], i-[24], j-[19], k-[20], l-[21], m-[26]$,

$n-[27], o-[28], p-[29]$.

references).

Overabundances of carbon and heavy-metal [s/Fe] overabundance (or lack thereof) in the atmosphere of the central star were published earlier by [19, 22, 25, 33] for IRAS04296+3429, 07134+1005, 08005-2356, and 23304+6147 respectively; [21] for IRAS 20000+3239; [23] and [34] for RAFGL 2688; [27] for V448 Lac and [28] for V354 Lac. Chemical abundances for five stars from Table 1 were also published by [35]. As follows from Table 2, all stars with excess carbon and heavy metals are objects with moderate iron deficiency and may belong to the thick disk of the Galaxy. 
The spectra of protoplanetary nebulae with F-K-type supergiant as central stars that have carbonenriched atmospheres show features of carbon-containing molecules $\mathrm{C}_{2}, \mathrm{C}_{3}, \mathrm{CN}$, and $\mathrm{CH}^{+}$. Position measurements of molecular features in the spectra indicate that they form in expanding circumstellar envelopes. Authors [18] and [19, 21, 22, 24, 26-29] used high-resolution optical spectra to analyze molecular bands for several post-AGB stars including some objects from Table 1.

It appears that the emission in the Swan bands or Na I D-lines is observed in the spectra of PPNe with bright and conspicuously asymmetric circumstellar nebulae. The results of spectroscopic observations of several PPNe confirm this hypothesis. An analysis of the spectra taken with the $6-\mathrm{m}$ telescope revealed $\mathrm{C}_{2}$ Swan emission bands of different intensities (relative to the continuum) in the spectra of the central stars of the following sources: IRAS 04296+3429 [22], 08005-2356 [19], RAFGL 2688 [26], IRAS 22223+4327 [27], and IRAS 23304+6147 [29, 33]. According to HST images [3, 9], these objects have structured (and often bipolar) envelopes.

In addition to a sample of related $\mathrm{C}$-rich objects with the above features, Table 1 also includes the infrared source IRAS 08005-2356. This object has so far been poorly studied, and no data are available either on the peculiarities of the chemical composition of its atmosphere or on the presence of the $21-\mu \mathrm{m}$ band. However, IRAS 08005-2356 can be viewed as related to the objects of this sample because its optical spectrum exhibits $\mathrm{C}_{2}$ Swan bands, the hydrogen and metal lines in its spectrum have emission-absorption profiles [19], and the circumstellar envelope is observed in CO emission [12].

Note that our subsample of PPNe, thoroughly studied by high-resolution spectra (Table 1), practically coincides with the list of $\mathrm{C}$-rich and $21 \mu \mathrm{m}$ protoplanetary nebulae the photometric and spectral properties of which were extensively studied in papers $[31,36]$. Of fundamental importance to us is the conclusion in [37] about the rare occurrence of binaries among post-AGB stars of the type considered, which is based on the long-term investigation of the velocity field in PPN atmospheres conducted by the above authors. Thus, the results of $[31,36,37]$ provide further evidence for the homogeneity of the PPNe subsample considered here.

\subsection{Peculiar metal absorptions in the optical spectra of selected post-AGB stars}

The systematic monitoring of PPN candidates, which we performed with a high spectral resolution, has released a new result - an unknown earlier peculiarity: the splitting (or asymmetry) of strongest metal absorptions due to distortion by envelope features. Now such peculiar profiles of the strongest absorptions 


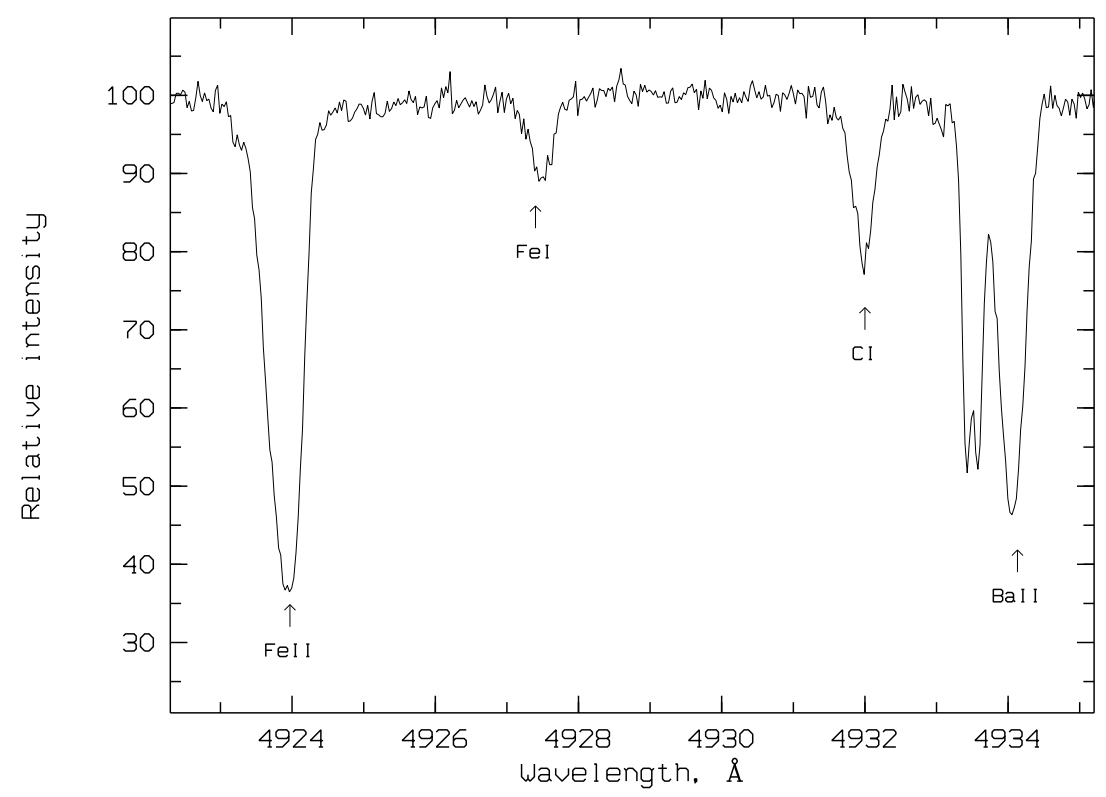

Figure 1. Fragment of the spectrum for V5112 Sgr containing split Ba II $4934 \AA$ line in the July 7, 2001 spectrum. The identification of main absorptions is indicated.

were revealed in the spectra of five stars listed in Table 2: CY CMi [24], V354 Lac [28, 38], V448 Lac [27], V5112 Sgr [20] and CGCS 6918 (IRAS 23304+6147) [29]. Let us illustrate this effect using the example of the spectrum of the high-latitude supergiant V5112 Sgr, where it is most pronounced. Figure 1 shows a fragment of the spectrum of V5112 Sgr taken on July 7, 2001 with a split Ba II $4934 \AA$ line. At the same time, the profiles of strong absorptions of iron-group metals in the spectrum of this star are neither asymmetric nor split, as is immediately apparent from the same Fig. 1, where we see a very strong but unsplit Fe II $4924 \AA$ line.

Besides, a comparison of the line profiles in the spectra of V5112 Sgr taken during different nights reveals substantial variability of the profile shapes and of the positions of the components of the split lines. To illustrate the variability effect, we show in Fig. 2 the Ba II $4934 \AA$ line profile, which is most asymmetric and most variable. The different widths of the components are immediately apparent in both Figs. 1 and 2: the red component is about twice broader than the blue components, which are offset substantially relative to the systemic velocity. This difference between the component widths indicates that the red and blue components form under different physical conditions. It follows also from Fig. 1 that the position of the photospheric (red) component of the complex profile is variable, whereas the blue components, which, as shown in [20], form in the envelope, are stable.

The semiregular variable V354 Lac is the closest analog to V5112 Sgr among the objects listed in Tables 1 


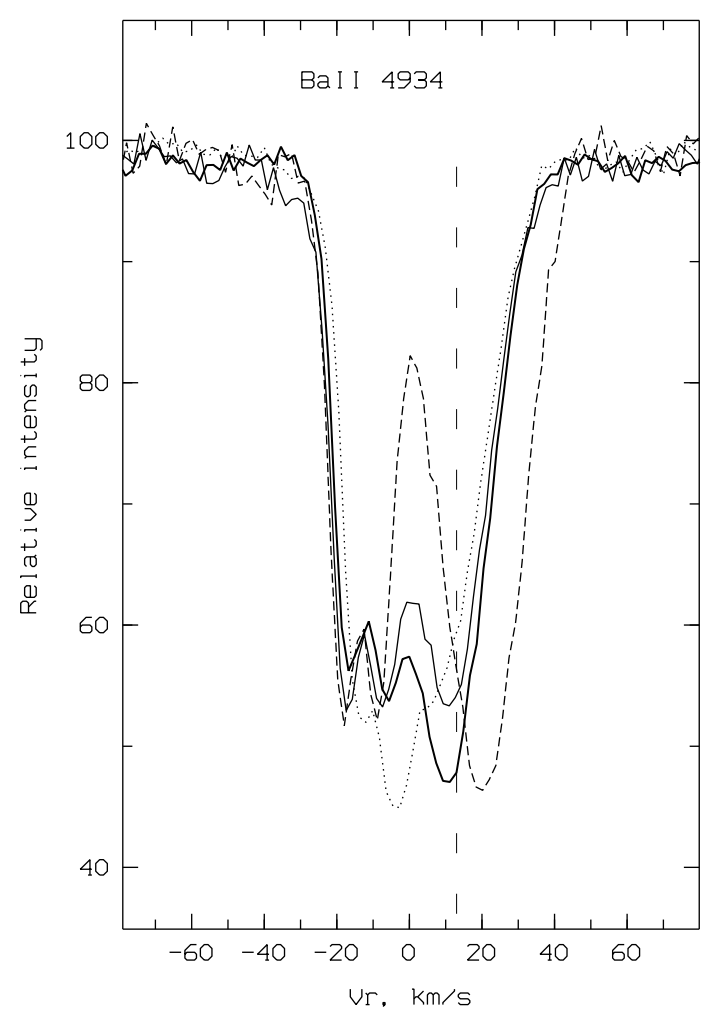

Figure 2. Variability of the Ba II 4934 A line profile in the spectra of V5112 Sgr taken in different years: August 2, 2012 (the thin solid line); June 13, 2011 (the solid bold line); August 14, 2006 (the dotted line); July 7, 2001 (the dashed line) $[20]$.

Table 2. Main atmospheric parameters of post-AGB stars with peculiar profiles of strongest absorptions: effective temperature, Teff, metallicity, $[\mathrm{Fe} / \mathrm{H}]_{\odot}$, and the mean excess of heavy metals synthesized in s-process, $[\mathrm{s} / \mathrm{Fe}]_{\odot}$

\begin{tabular}{|c|c|c|c|c|c|}
\hline Star & $I R A S$ & \multicolumn{4}{|c|}{ Teff, $K[\mathrm{Fe} / \mathrm{H}]_{\odot}[\mathrm{s} / \mathrm{Fe}]_{\odot} \operatorname{Ref}$} \\
\hline CY CMi & $7134+1005$ & 7000 & -1.0 & +1.4 & a \\
\hline V5112 Sgr & $9500-1709$ & 8000 & -0.6 & +1.1 & $\mathrm{~b}$ \\
\hline V448 Lac & $22223+4327$ & 6500 & -0.3 & +0.9 & b \\
\hline V354 Lac & $22272+5435$ & 5650 & -0.8 & +1.2 & $\mathrm{c}$ \\
\hline CGCS 6918 & $3304+6147$ & 5900 & -0.6 & +1.2 & d \\
\hline
\end{tabular}

$a-[25], b-[35], c-[28], d-[33]$.

and 2 in terms of the structured envelope and chemical abundances. The spectroscopic monitoring of this star [28] carried out at the SAO 6-m telescope with a resolution of $R=60000$ also revealed the splitting of the strongest absorptions with the low-level excitation potential of $\chi_{\text {low }} \leq 1 \mathrm{eV}$. An analysis of the kinematic pattern showed that the blue component of the split line forms in the powerful gas and dust envelope of V354 Lac. This splitting shows up most conspicuously in the profile of the strong Ba II $6141 \AA$ 


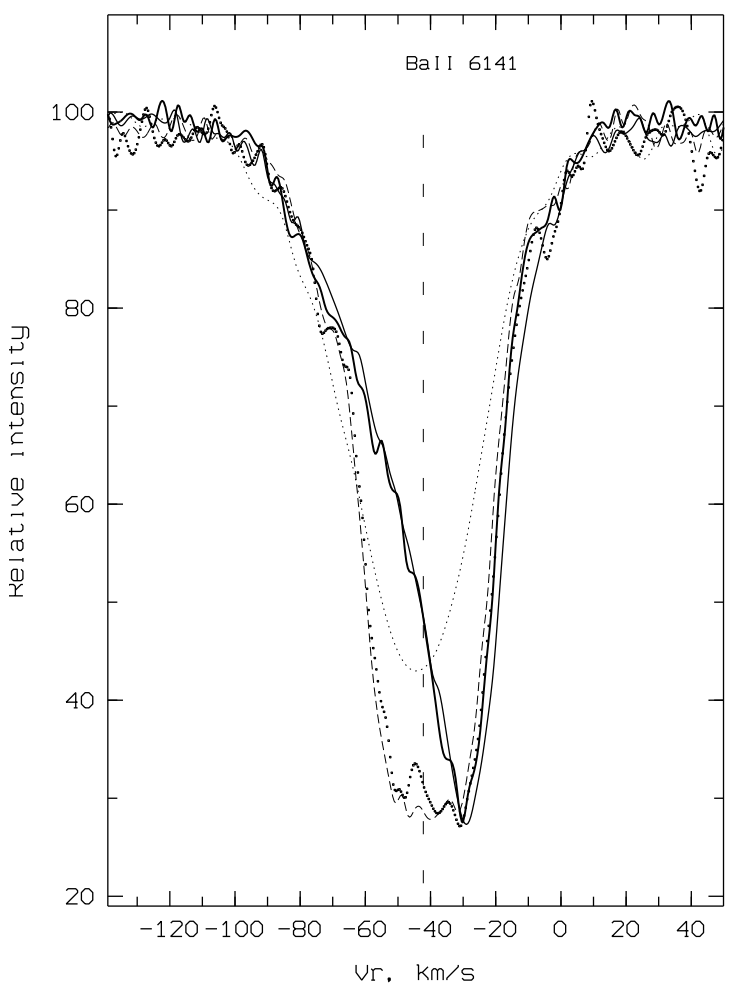

Figure 3. Profile variations of the BaII 6141 A line in the spectra of V448 Lac at various epochs: JD 2454760.17 (bold), JD 2454721.15 (thin), JD 2453694.36 (dashed), and JD 2452131.53 (dotted)

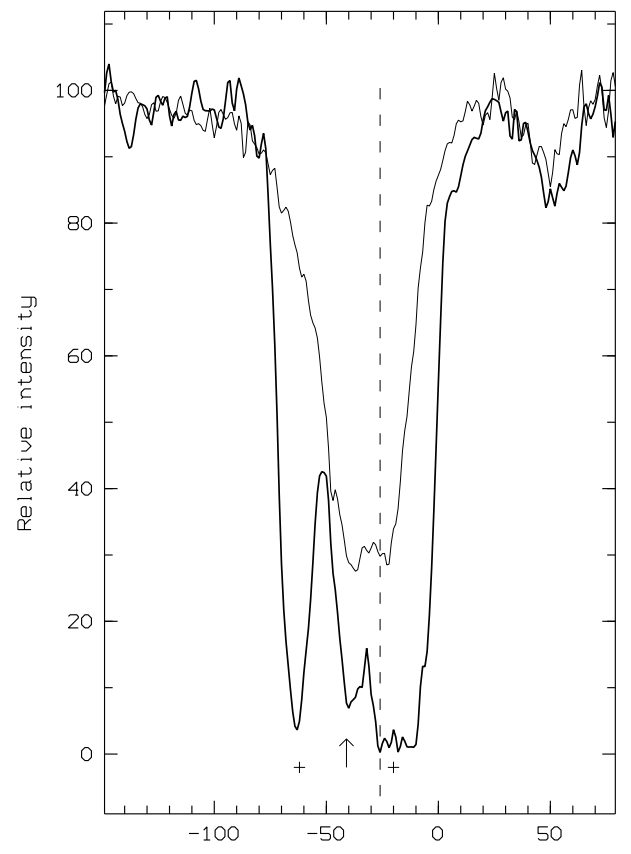

Figure 4. Profiles of the split lines in the October 12, 2013 spectrum of CGCS 6918: $\mathrm{D}_{2} \mathrm{NaI}$ - the bold line and Ba II 6141 - the the thin line 
line. The shift of the blue component of the Ba II line coincides with that of the circumstellar component of $\mathrm{Na} I \mathrm{D}$ lines, which forms in the same layers as the circumstellar $\mathrm{C}_{2}$ Swan bands. This coincidence indicates that the complex profile of the BaII $6141 \AA$ line contains, in addition to the atmospheric component, a component that forms in the circumstellar envelope. Such splitting (or the profile asymmetry due to the more shallow slope of the blue wing) is also observed for other BaII $(\lambda \lambda 4554,5853,6496 \AA)$ lines as well as for the strong Y II $5402 \AA$, La II $6390 \AA$, and Nd II 5234, $5293 \AA$ lines. The lines of these ions in the spectrum of V354 Lac are enhanced to the extent that their intensities are comparable to those of neutral-hydrogen lines. For example, the equivalent width of the Ba II $6141 \AA$ line reaches $W_{\lambda} \approx 1 \AA$, and that of $\mathrm{H} \beta$ reaches $W_{\lambda} \approx 2.5 \AA$.

As follows from Figs. 3 and 4, in the spectra of two stars-V448 Lac and CGCS 6918 - a central star of IRAS23304, the Ba II $6141 \AA$ line profile is or asymmetric or split for different moments of observations. Note that the variations are displayed only by the short-wavelength profile wings and the position of the core, whereas the positions and intensities of the long-wavelength wings of these strong Ba II, La II, and Y II absorption lines do not vary in time.

Asymmetric and variable profiles of strongest lines (Y II, Ba II, Fe II and other strong absorption lines) formed in the expanding stellar atmosphere were also found in [13] in the spectra of CY CMi. However, as follows from Fig. 5 in the paper by these authors, in the spectra of CY CMi profiles are just only asymmetric, but not split. Thus, we see a difference between the peculiarity types of the profiles of three stars-V5112 Sgr, V354 Lac, and CGCS 6918 with split profiles of the strongest absorptions of selected elements, and two stars-CY CMi and V448 Lac with asymmetric but unsplit profiles. Such a difference permit us to suggest that the morphology of the circumstellar envelope may be the main factor that causes the peculiarity and variability of the profiles of the strongest lines. As is evident from Table 1, the two stars V5112 Sgr, V354 Lac and CGCS 6918 with split absorptions have bipolar (or quadrupolar) envelopes, whereas the absorptions are unsplit in the spectra of CY CMi and V448 Lac the envelope of these stars have a less structured environment. This hypothesis is further corroborated by the three-component structure of the strong absorption profiles observed in the spectrum of V5112 Sgr, where CO-observations show both the slow $\left(\mathrm{V}_{\exp }=10 \mathrm{~km} \mathrm{~s}^{-1}\right)$ and the fast $\left(30-40 \mathrm{~km} \mathrm{~s}^{-1}\right)$ expansion [14]. As follows from data in Table 1, the profiles of the split lines include a photospheric component and two envelope components, one of which, like in the case of the CO-profile, arises in the envelope that formed at the AGB-stage and expands at a velocity of $\mathrm{V}_{\exp }(2) \approx 20 \mathrm{kms}^{-1}$, and the other one arises in the envelope that moves at a velocity of $\mathrm{V}_{\exp }(1) \approx 30 \mathrm{kms}^{-1}$ and formed later.

Worth mentioning here is another interesting phenomenon which was revealed in the spectrum of 
V5112 Sgr. Klochkova [20] showed that the optical spectrum of this high latitude post-AGB supergiant contains weak absorptions whose positions are indicative of their formation in the circumstellar envelope. The velocities $\mathrm{V}_{r}(\mathrm{DB})$ were measured in the 5780-6379 $\AA$ wavelength interval from the positions of reliably identified 5780, 5797, 6196, 6234, and $6379 \AA$ features. The mean $\mathrm{V}_{r}(\mathrm{DIB})$ averaged over several spectra and determined with an accuracy better than $\pm 0.5 \mathrm{~km} \mathrm{~s}^{-1}$ agrees excellently with the velocity determined from the blue component of the Na I D-lines. The resulting agreement leads us to conclude that the weak bands found in the spectrum of V5112 Sgr origin in the circumstellar envelope. Kipper [39] independently came to similar conclusions for the same object based on the spectra of V5112 Sgr taken with a different instrument. This DIBs identification at the present time is a unique result in the task of searching for these spectral features in the circumstellar environment.

\section{DISCUSSION OF THE RESULTS}

Note that the splitting of strong absorptions were observed in the spectra only those post-AGB stars that are listed in the Table 2 , in atmospheres which were found significant excess carbon and heavy metals. The second precondition for the splitting of the strong absorptions in the spectrum of these post-AGB stars is presence of a complex structured envelope. We can suggest that the process of the formation of a circumstellar envelope may contribute to the enrichment of this envelope by products of stellar nucleosynthesis. The profiles of the split lines contain a photospheric and one-two envelope components, one of which, like in the case of the $\mathrm{CO}$-profile, arises in the envelope that formed at the AGB-stage, and the other one-in the envelope that formed later. The circumstellar components of strong heavymetal absorptions have been conclusively identified in the spectra of V5112 Sgr [20], V354 Lac [28] and CGCS 6918 [29].

The structure of the circumstellar nebulae of V354 Lac and CGCS 6918 may be more complex than it appears in the HST observations (see Table 1). Polarimetric observations of V354 Lac [40] indicate the presence of a ring structure embedded in an extended nebula. Nakashima et al. [41] point out that the axes of the optical and infrared images of the nebula are almost perpendicular to each other. Based on the kinematic pattern of the nebula as determined from the mapping of the CO-emission, authors [41] concluded that the structure of the nebula includes not only a torus and a spherical component but also another element (possibly a jet).

Currently, no consensus has been reached concerning the development of deviations from spherical symmetry in PPNe. Authors [3, 9] analyzed high spatial resolution optical images of a sample of PPNe 
taken by the Hubble Space Telescope and concluded that the optical depth of the circumstellar matter is the crucial factor that determines the formation of a particular morphology of stellar envelopes. The dense and often spherical envelope that formed during the AGB-stage is believed to expand slowly, whereas the rapidly expanding feature is the axisymmetric part of the envelope that formed later at the post-AGB stage [42]. The sequence of these processes results in the development of an optical depth gradient in the direction from the equator to the polar axis of the system. The presence of a companion and/or a magnetic field in the system may also prove to be the physical factor that causes the loss of the spherical symmetry of the stellar envelope during the short evolutionary interval between the AGB and post-AGB stages (see [43, 44] and references therein). In their recent paper [45] proposed a simple PPN model based on a pair of evacuated cavities inside a dense spherical halo. The above authors demonstrated that all the morphological features observed in real bipolar PPNe can be reproduced by varying the available parameters (mass density inside the cavity, its size and orientation) of this model.

So far, the discovery of the heavy-metal enrichment of the circumstellar envelopes of the post-AGB supergiants V5112 Sgr, V354 Lac and CGCS 6918 remain the only results. Here it should be recalled that Mauron \& Huggins [46] detected atomic metals (Ca, Fe and upper limits for Al, Ti, Mn and Sr) in the C-rich circumstellar envelope of the AGB-star CW Leo, which is a central star of the IR source IRC +10216 .

The effect of splitting of metallic lines found requires to continue the high resolution spectroscopy of very related post-AGB stars. The most promising objects could be IRAS 04296+3429 and RAFGL 2688 . It follows from Table 1 that the totality of properties of their envelopes coincide with those of the objects listed in Table 2: they are $\mathrm{C}$-enriched and have a very complex structure. Besides, the atmospheres of the weak central stars of both sources are enriched in heavy metals [22, 26].

Evidently, the next step in study of the discovered splitting of the profiles should be theoretical modeling of the spectra envelopes and calculation chemical abundances, which could be consider with regard to selective depletion of chemical elements in a dusty environment.

\section{MAIN COCLUSIONS}

We used the results of high spectral resolution observations made with the $6-\mathrm{m}$ telescope to analyze the peculiarities of the optical spectra of a sample of post-AGB stars with atmospheres enriched in carbon and heavy s-process metals and with carbon-enriched circumstellar envelopes. 
We showed that presence of the peculiarities of the line profiles (the asymmetry and splitting of the profiles of strong absorptions) is associated with the kinematic and chemical properties of the circumstellar envelope and the type of its morphology. The splitting of the profiles of the strongest heavy-metal absorptions in the spectra of the V5112 Sgr, V354 Lac and CGCS 6918 supergiants found as a result of our observations suggests that the formation of a structured circumstellar envelope is accompanied by the enrichment of this envelope with the products of stellar nucleosynthesis.

Attempts to find a definite link between the peculiarities of the optical spectrum and the morphology of the circumstellar environment are complicated by the fact that the observed structure of the envelope depends strongly on the inclination of the symmetry axis to the line of sight and on the angular resolution of the spectroscopic and direct-imaging instruments.

In the spectrum of the high latitude post-AGB supergiant V5112 Sgr were revealed weak absorptional DIBs 5780, 5797, 6196, 6234, and $6379 \AA$. Their mean radial velocity $\mathrm{V}_{r}$ (DIBs) determined with an accuracy better than $\pm 0.5 \mathrm{~km} \mathrm{~s}^{-1}$ agrees excellently with the velocity determined from the blue circumstellar component of the NaI D-lines.

\section{ACKNOWLEDGMENTS}

This work was supported by the Russian Foundation for Basic Research (project No. 14-02-00291 a). This research has made use of the SIMBAD database, operated at CDS, Strasbourg, France, and NASA's Astrophysics Data System.

1. Herwig F., 2005, ARA\&A, 43, 435

2. Käppeler F., Gallino R., Bisterzo S., Aoki W., 2011, Rev. Mod. Phys. 83, 157

3. Siódmiak N., Meixner M., Ueta T., et al., 2008, ApJ, 677, 382

4. Lagadec E., Verhoelst V., Mékarnia D., et al., 2011, MNRAS, 417, 32

5. Bieging J.H., Schmidt G.D., Smith P.S., and Oppenheimer B.D., 2006, ApJ, 639, 1053

6. Panchuk V., Klochkova V, Yushkin M., and Najdenov I., 2009, J. Optical Technology, 76, 87

7. Panchuk V.E., Najdenov I.D., Klochkova V.G., et al., 1998, Astrophys. Bull. 44, 127

8. Klochkova V.G. Astrophys. Bull., 2014, 69, 279

9. Ueta T., Meixner M., Bobrowsky M., 2000, ApJ, 528, 861 
10. Sahai R., Morris M., Sánchez Contreras C., Claussen M., 2007, AJ, 134, 2200

11. Hrivnak B.J., Bieging J.H., 2005 ApJ, 624, 331

12. Hu J.Y., te Lintel Hekkert P., Slijkhuis S., et al., 1994, A\&AS, 103, 301

13. Klochkova V.G., Chentsov E.L. , 2007, Astron. Rep. 51, 994

14. Bujarrabal V., Alcolea J., and Planesas P., 1992, A\&A, 257, 701

15. Omont A., Loup C., Forveille T., et al., 1993, A\&A, 267, 515

16. Loup C., Forveille T., Omont A., Paul J.P., 1993, A\&AS, 99, 291

17. Klochkova V.G. Astrophys. Bull., 2012, 67, 385

18. Bakker E.J., van Dishoeck E.F., Waters L.B.F.M., Schoenmaker T., 1997, A\&A, 323, 469

19. Klochkova V.G., Chentsov E.L., 2004, Astron. Rep. 48, 301

20. Klochkova V.G., 2013, Astron. Lett., 39, 765

21. Klochkova V.G. and Kipper T., 2006, Baltic Astronomy 15, 395

22. Klochkova V.G., Szczerba R., Panchuk V.E., and Volk K., 1999, A\&A, 345, 905

23. Klochkova V.G., Panchuk V.E., Yushkin M.V., Miroshnichenko A.S., 2004, Astron. Rep. 48, 288

24. Klochkova V.G., Chentsov E.L., Tavolganskaya N.S., Shapovalov M.V., 2007, Astrophys. Bull. 62, 162

25. Klochkova V.G., 1995, MNRAS, 272, 710

26. Klochkova V.G., Szczerba R., and Panchuk V.E., 2000a, Astron. Let. 26, 439

27. Klochkova V.G., Panchuk V.E., Tavolzhanskaya N.S., 2010, Astron. Rep. 54, 234

28. Klochkova V.G., Panchuk V.E., Tavolganskaya N.S., 2009, Astrophys. Bull. 64, 155

29. Klochkova V.G., Panchuk V.E., Tavolganskaya N.S. 2015, Astron. Let. 41, 14

30. Kwok S., Volk K., and Hrivnak B.J., 1999, IAU Symp., No. 191, 297

31. Hrivnak B.J., Volk K., Kwok S., 2009, ApJ, 694, 1147

32. Li A., Liu J.M., Jiang B.W., 2013, ApJ, 777, 111

33. Klochkova V.G., Szczerba R., and Panchuk V.E., 2000b, Astron. Let. 26, 88

34. Ishigaki M.N., Parthasarathy M., Reddy B.E., et al., 2012, MNRAS, 425, 997

35. van Winckel H., Reyniers M., 2000, A\&A, 354, 135

36. Hrivnak B.J., Lu W., Maupin R.E., Spitzbart B.D., 2010, ApJ, 709, 1042

37. Hrivnak B.J., Lu W., Wefel K.L., et al., 2011, ApJ, 734, 25

38. Klochkova V.G., 2009, Astron. Let. 35, 457

39. Kipper T., 2013, Baltic Astronomy, 22, 297

40. Gledhill T.M., Chrysostomou A., Hough J.H, Yates J.A., 2001, MNRAS, 322, 321 
41. Nakashima J., Koning N., Volgenau N.H., et al., 2012, ApJ, 759, 61

42. Castro-Carrizo A., Quintana-Lacaci G., Neri R., et al., 2010, A\&A, 523, A59

43. Huggins P.J., Mauron N., Wirth E.A., 2009, MNRAS, 396, 1805

44. Leal-Ferreira M.L., Vlemmings W.H.T., Diamond P.J., et al., 2012, A\&A, 540, A42

45. Koning N., Kwok S., Steffen W., 2013, ApJ, 765, 92

46. Mauron N., Huggins P.J., 2010, A\&A, 513, A31 